\title{
State Tomography of Capacitively Shunted Phase Qubits with High Fidelity
}

\author{
Matthias Steffen, M. Ansmann, R. McDermott, N. Katz, Radoslaw C. Bialczak, Erik Lucero, \\ Matthew Neeley, E. M. Weig, A. N. Cleland, and John M. Martinis* \\ Department of Physics and California Nanosystems Institute, University of California, Santa Barbara, California 93106, USA
} (Received 17 February 2006; published 2 August 2006)

\begin{abstract}
We introduce a new design concept for superconducting phase quantum bits (qubits) in which we explicitly separate the capacitive element from the Josephson tunnel junction for improved qubit performance. The number of two-level systems that couple to the qubit is thereby reduced by an order of magnitude and the measurement fidelity improves to $90 \%$. This improved design enables the first demonstration of quantum state tomography with superconducting qubits using single-shot measurements.
\end{abstract}

DOI: 10.1103/PhysRevLett.97.050502

PACS numbers: 03.67.Lx, 74.50.+r, 85.25.Am

Superconducting circuits using Josephson junctions provide a promising approach towards the construction of a scalable solid-state quantum computer [1]. The phase qubit [2] has significant potential because it is tunable and coupled qubits have been measured simultaneously [3]. However, progress using phase qubits has been hindered by a large density of two-level system (TLS) defects residing in the tunnel junction that couple to the qubit [4], resulting in avoided level crossings (splittings) in the qubit spectroscopy, as shown in Fig. 1. These TLS resonances degrade measurement fidelity [5] and couple to the qubit state even when off resonance, reducing the amplitude of the qubit coherence $[4,6]$. In combination, these effects prevent implementation of high-fidelity quantum gates. In recent work these splittings were shown to correspond to dielectric loss, and improving the dielectric of the tunnel junction was conjectured as one approach to increase the coherence of phase qubits [6].

Here we introduce and implement a new circuit layout that reduces the number of splittings in the qubit spectroscopy without new materials engineering. A dramatic improvement in the measurement fidelity is observed [5], enabling the first demonstration of quantum state tomography using a superconducting qubit with single-shot measurements [7]. These results place the phase qubit in position for even more complex two-qubit quantum gates.

Loss of measurement fidelity from TLS depends on both the density and size of the splittings, and scales as $A_{J} / C$, where $A_{J}$ is the tunnel junction area and $C$ is the total capacitance $[5,6]$. Similarly, loss of qubit coherence from off-resonant coupling scales as $A_{J}^{2} / C[6,8]$. The standard (single-element) design [5,6] has $C \propto A_{J}$, and the measurement and coherence loss scale as $\left(A_{J}\right)^{0}$ and $A_{J}$, respectively. However, improved scaling can be achieved by separating the tunnel junction from its capacitance by adding an external low-loss capacitor while keeping the junction's critical current constant. In this case, the losses scale as $A_{J}$ and $A_{J}^{2}$, respectively. By reducing the area of the tunnel junction from $\sim 10 \mu \mathrm{m}^{2}$ to $\sim 1 \mu \mathrm{m}^{2}$, we expect a decrease in the measurement errors by a factor of 10 and an increase in the qubit coherence amplitude by a factor of 100. The resulting qubit design of Fig. 2(a)-2(c) therefore simply substitutes the capacitance from the tunnel junction with an external element.

The redesign requires reliable fabrication of tunnel junctions with an area of about $\sim 1 \mu \mathrm{m}^{2}$. The tunnel barrier is formed by an Ar ion-mill clean followed by thermal oxidation of the $\mathrm{Al}$ base electrode and blanket evaporation of the counterelectrode. The Josephson junction is next defined by optical lithography and a reactive ion etch of the counterelectrode in an $\mathrm{Ar} / \mathrm{Cl}_{2}$ plasma. A micrograph of the resulting small-area junction is shown in Fig. 2(b). The

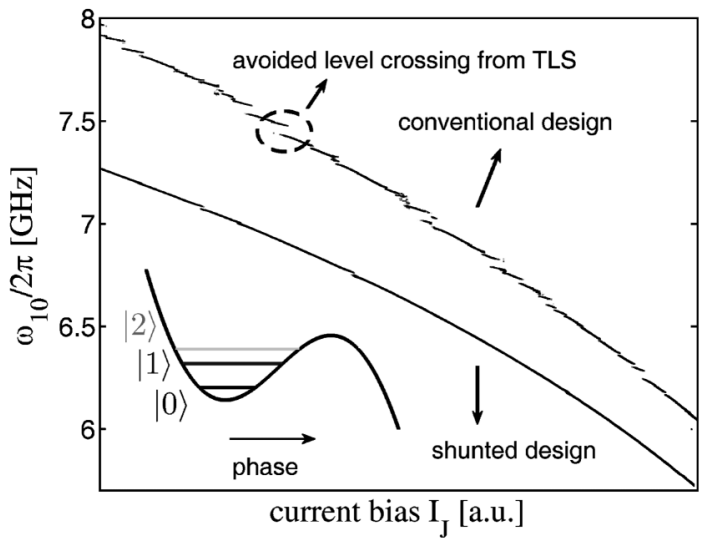

FIG. 1. Qubit frequency $\omega_{10} / 2 \pi$ vs current bias for the conventional single-element design (top trace, shifted for clarity) and the capacitively shunted design (bottom trace). The bias current $I_{J}$ tunes the frequency of the qubit $[4,5]$. The density of splittings in the conventional design is high enough that the qubit is almost always coupled to at least one TLS. The state occupation of $|1\rangle$ is consequently diminished, giving rise to decoherence and thus reducing qubit performance. The new design has about one tenth of the area and consequently exhibits a reduction in the number of avoided level crossings by approximately a factor of 10. The inset sketches the potential energy vs phase difference across the tunnel junction as well as the first three energy levels, $|0\rangle,|1\rangle$, and $|2\rangle$. The resonance frequency $\omega_{10} / 2 \pi$ differs from $\omega_{21} / 2 \pi$ by a few percent. 

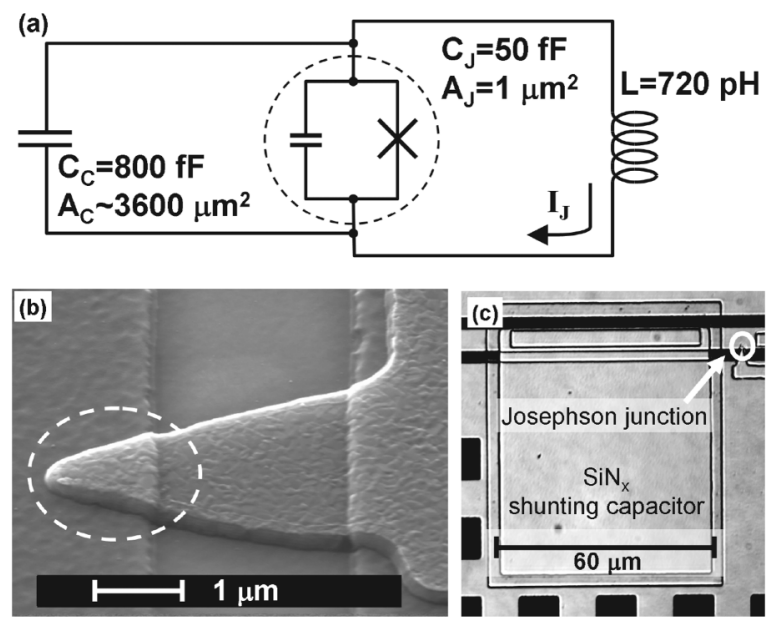

FIG. 2. Circuit diagram and micrograph of the redesigned phase qubit. (a) A small-area $\left(A_{J} \sim 1 \mu \mathrm{m}^{2}\right)$ Josephson junction with little self-capacitance $\left(C_{J}=50 \mathrm{fF}\right)$ is shunted by a large $\left(A_{C} \sim 3600 \mu \mathrm{m}^{2}\right)$ high quality capacitor with $C_{C}=800 \mathrm{fF}$. The qubit bias current $I_{J}$ is induced through an inductor with $L=$ $720 \mathrm{pH}$. (b) A scanning electron microscope image of the smallarea Josephson junction shows well-defined features at submicron length scales. (c) An optical image of the shunting capacitor and the Josephson junction (white circle).

shunting capacitor, shown in Fig. 2(c), is made of silicon nitride. It was previously shown to have 20 times lower dielectric loss than $\mathrm{SiO}_{2}$ [6] in our system, and thus is an acceptable choice for this first demonstration.

Experimental tests $[4,5]$ on the redesigned phase qubit confirm the expected behavior. The number of splittings visible in the qubit spectroscopy is reduced roughly by an order of magnitude, yet their sizes are comparable to those of the conventional design (see Fig. 1). The visibility of the Rabi oscillations [Fig. 3(a)] is about $85 \%$ and the decay is limited by the measured energy relaxation time of $T 1 \approx$ 110 ns. Because of the small number of splittings, we are able to decrease the Rabi oscillation period to about $12.5 \mathrm{~ns}$ [limited by pulse shaping and the detuning of $\omega_{21}$ [9]], compared to a $\sim 50$ ns Rabi oscillation period using the conventional design [6].

From the data, we extract a measurement fidelity that is close to $90 \%$, defined as the measured signal following the application of a $\pi$ pulse, which populates the $|1\rangle$ state. This is an improvement of about $40 \%$ compared with the measurement fidelity using the standard phase qubit design. Our observations confirm the prediction that measurement fidelity is reduced by sweeping through avoided level crossings during our measurement pulse [5]. Half of the remaining $10 \%$ loss in the measurement fidelity can be attributed to energy relaxation (as the measurement takes about $5 \mathrm{~ns}$ ), while the remaining half can be attributed to a loss in signal due to sweeping through a few remaining junction resonances. Additional experimental data, including Ramsey fringes and a spin echo [Fig. 3(b) and 3(c)], are consistent with measured linewidths from the qubit spectroscopy as well as the energy relaxation time. (a)

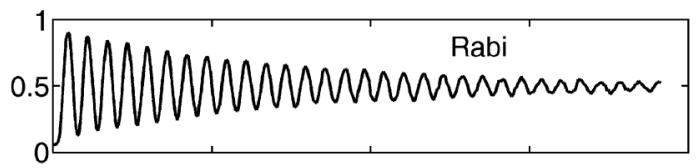

(b)

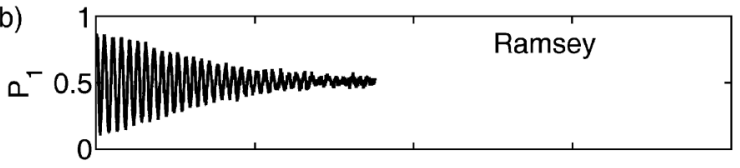

(c)

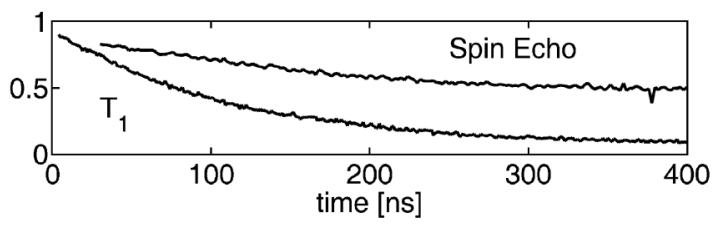

FIG. 3. Experimental results using the capacitively shunted phase qubit design. The vertical axis of each plot displays the probability $P_{1}$ of occupying the $|1\rangle$ state. All results are obtained at a bias where $\omega_{10} / 2 \pi=5.838 \mathrm{GHz}$. (a) Rabi oscillations have a visibility of about $85 \%$ and decay at a rate consistent with the measured qubit relaxation time of $T_{1} \approx 110 \mathrm{~ns}$ [shown in (c)]. (b) The Ramsey fringes (detuning of $196 \mathrm{MHz}$ ) decay nonexponentially and are consistent with spectroscopic linewidths. We extract a dephasing time of $T_{2}^{*} \approx 90 \mathrm{~ns}$. The two $90^{\circ}$ rotations used in the experiment were $4 \mathrm{~ns}$ long. (c) The impact of lowfrequency $1 / f$ noise can be significantly reduced using a spin echo sequence. We extract an intrinsic dephasing time of $T_{2} \approx$ $160 \mathrm{~ns}$. The duration of the two $90^{\circ}$ and the $180^{\circ}$ rotations was 5 and $8 \mathrm{~ns}$, respectively. Measurement of the energy decay $\left(T_{1}\right)$ is also plotted. The pulse duration was $6 \mathrm{~ns}$.

The energy relaxation of the qubit is limited by the dielectric loss of the $\mathrm{SiN}_{x}$ shunting capacitor, which has a measured loss tangent of $\delta_{i} \approx 1.5 \times 10^{-4}$. With a qubit frequency $\omega_{10} / 2 \pi \approx 6 \mathrm{GHz}$, we expect an energy relaxation of $T_{1}=1 / \delta_{i} \omega^{10} \approx 170 \mathrm{~ns}$, which is close to the measured value. Further improvements in $T_{1}$ are possible by simply fabricating shunting capacitors with even lower loss tangents, highlighting the fact that the decoherence from splittings and energy relaxation can be separately optimized.

The demonstrated improvements in fidelity now enable state tomography, which is necessary for a full characterization of qubit states and gate operations. Typically, state tomography involves measuring an unknown quantum state in different basis sets to extract its location on the Bloch sphere. However, in our experiment it is more convenient to always measure in the $|0\rangle$ and $|1\rangle$ basis (positive and negative $\hat{z}$ directions on the Bloch sphere), and perform the basis change through single qubit rotations prior to measurement, as discussed below.

To perform tomography, we have designed and built a custom microwave pulse sequencer capable of producing microwave pulses with arbitrary amplitude and phase (see Fig. 4). We use an $I Q$ mixer that adds, with separate amplitude control (11 bit resolution each, updated every $8 \mathrm{~ns}$ ), the two quadrature components $I$ and $Q$ of a continuous-wave microwave signal, controlling the 


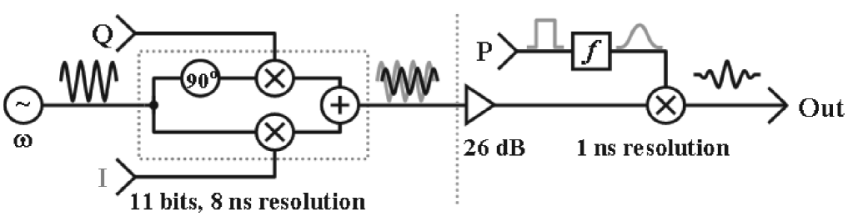

FIG. 4. Schematic diagram of the microwave pulser. The amplitudes of the two quadrature components $I$ and $Q$ are added to obtain microwaves with adjustable amplitude and phase, and are then amplified by $26 \mathrm{~dB}$. A filtered digital pulse is then used in a second mixer to produce a Gaussian-shaped microwave pulse.

amount of rotation about the $\hat{y}$ and $\hat{x}$ directions, respectively. A second mixer performs gating and pulse shaping with a time resolution of $1 \mathrm{~ns}$.

We use this microwave pulse sequencer to implement state tomography with two different techniques. The first method simply rotates an unknown Bloch vector over all rotation amplitudes and angles in the $x-y$ plane of the Bloch sphere, corresponding to sweeping over all accessible $I$ and $Q$ values, prior to measuring the final state occupation probability $P_{1}$ of $|1\rangle$. From the resulting twodimensional probability map, the direction of the Bloch vector can be simply computed from the amplitude and components of the digital-to-analog converter values at maximum $P_{1}$, and the Bloch vector length from the maximum contrast of $P_{1}$.

In Fig. 5 we plot $P_{1}$ as a function of amplitude of the $I$ and $Q$ components for several different initial states. In Fig. 5(a) the initial state is the ground state $|0\rangle$, and we observe generalized Rabi oscillations that are independent of the microwave phase, as expected. For the $|1\rangle$ state of Fig. 5(b), the populations are inverted as compared to Fig. 5(a). In Fig. 5(c) the initial state is the superposition $(|0\rangle+|1\rangle) / \sqrt{2}$, which is pointing along the $\hat{x}$ direction on the Bloch sphere. As a result, rotations about the $\hat{x}$ direction (controlled by $\mathrm{DAC}_{Q}$ ) will not affect the state. However, rotations along the $\hat{y}$ direction (controlled by $\mathrm{DAC}_{I}$ ) rotate the state and result in Rabi oscillations. The state $(|0\rangle+i|1\rangle) / \sqrt{2}$ is similarly plotted in Fig. 5(d), and shows a $90^{\circ}$ rotation in the direction of oscillations as compared to Fig. 5(c). Theoretical predictions are shown in the insets of Fig. 5; small differences arise primarily from off-resonance effects of the $|2\rangle$ state [9].

The data clearly show this technique can be used to reconstruct an unknown quantum state for complete testing of one qubit. However, a more efficient and widely used technique makes use of the fact that any arbitrary single qubit density matrix can be described by $\rho=(I+$ $\left.\sum_{i=1}^{3} c_{i} \sigma_{i}\right) / 2$, where $\sigma_{i}$ denotes the Pauli matrices, $I$ is the identity matrix, and $c_{i}$ are real coefficients [10,11]. Therefore, in order to reconstruct an unknown quantum state, we must identify the three-component vector $\vec{c}$.

The simplest approach measures the three components $c_{i}$ directly by performing three different readout schemes. The first readout is a simple measurement of the state
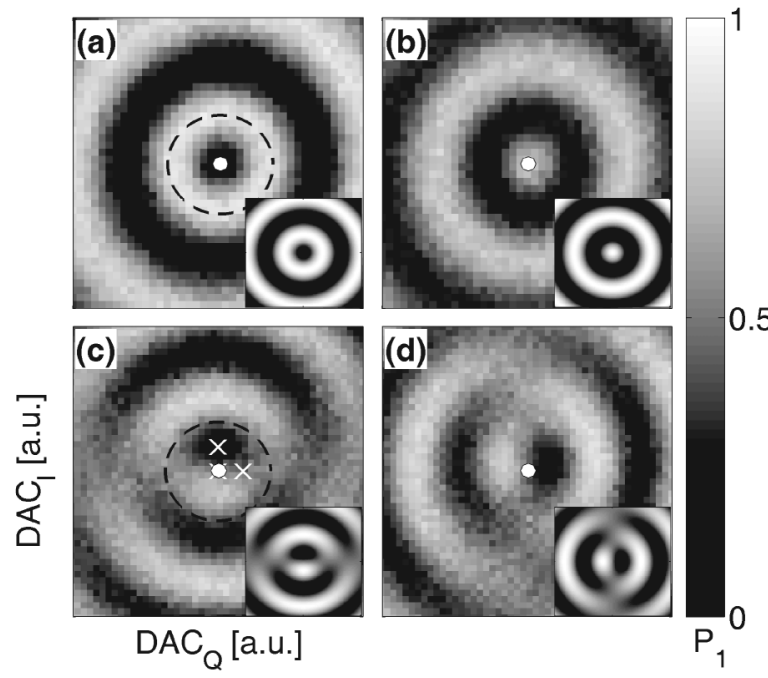

FIG. 5. Probability $P_{1}$ of occupying state $|1\rangle$ vs $\mathrm{DAC}_{I}$ and DAC $_{Q}$, which control the amplitude of the $\hat{y}$ and $\hat{x}$ tomography rotations, for the input states (a) $|0\rangle$, (b) $|1\rangle$, (c) $(|0\rangle+|1\rangle) / \sqrt{2}$, and $(\mathrm{d})(|0\rangle+i|1\rangle) / \sqrt{2}$. The tomography pulse was $16 \mathrm{~ns}$ long, and the state preparation pulses for the $90^{\circ}$ and $180^{\circ}$ rotations were 4 and $8 \mathrm{~ns}$ long, respectively. All experiments were performed on resonance with $\omega_{10} / 2 \pi=5.838 \mathrm{GHz}$, using 200 statistics. All axes have the same relative scale. The dashed black circle indicates a total pulse amplitude corresponding to a $\pi$ pulse at different phases. The white dots label the zero amplitude points, and the three white crosses indicate the measurements necessary for a simplified state tomography technique (used for Fig. 6). The inset in each plot shows the predicted probability map.

occupation probability of $|1\rangle$, which measures the $-\sigma_{z}$ component. The second (third) readout applies a $90^{\circ}$ rotation around the $\hat{x}$ direction ( $\hat{y}$ direction), followed by a measurement of the occupation probability of $|1\rangle$, measuring the $-\sigma_{y}\left(+\sigma_{x}\right)$ components. Using the three measured occupation probabilities we can immediately infer the quantum state and place it on the Bloch sphere. Experimentally, this technique is advantageous because it requires only three different readout sequences instead of acquiring a two-dimensional map.

We use this state tomography to trace out the time evolution of the single qubit quantum state in a Ramseytype experiment, as shown in Fig. 6(a). In this sequence, we include a current pulse during free evolution to rotate the qubit state about the $\hat{z}$ axis with a frequency of $37 \mathrm{MHz}$ relative to the rotating frame. This rotation can be inferred from the raw data [Fig. 6(b)] and can be clearly observed when reconstructing the Bloch vector [Fig. 6(c)]. Dephasing causes the $x y$ component to shrink [Fig. 6(c), left panel] and relax toward the ground state [Fig. 6(c), right panel]. These effects are confirmed by a theoretical model which includes energy relaxation and dephasing [see Fig. 6(d)]. The main difference between the experiment and theory is that the length of the reconstructed vector of the first point is about $80 \%$ instead of unity. 
(a)

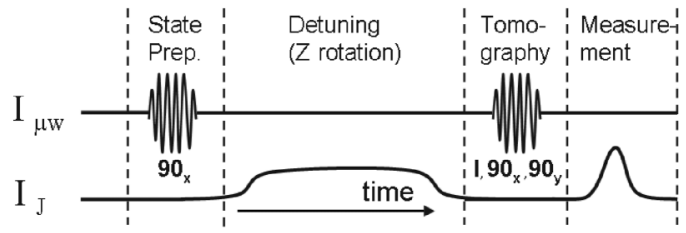

(b)
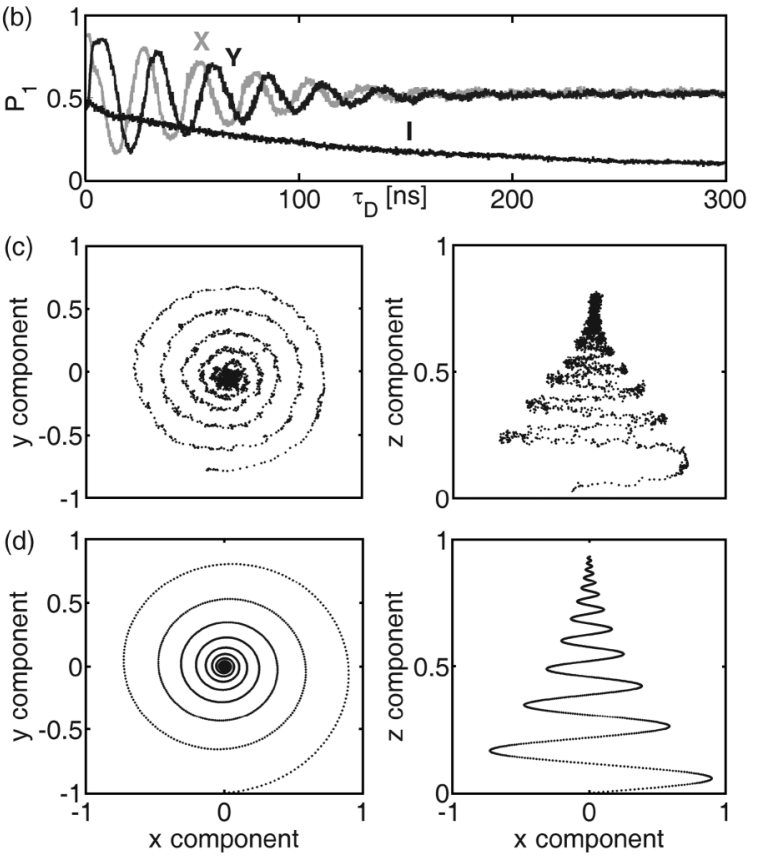

FIG. 6. State tomography of the Bloch vector during a Ramsey-type experiment. (a) Sequence of operations of the experiment. A resonant microwave pulse $I_{\mu \mathrm{m}}$ at frequency $\omega_{10} / 2 \pi=5.838 \mathrm{GHz}$ is first applied to give a $90^{\circ}$ rotation about the $\hat{x}$ axis. An adiabatic bias pulse $I_{J}$ with rise and fall times of abut $1 \mathrm{~ns}$ is then applied for a time $\tau_{d}$, during which the qubit is detuned by $37 \mathrm{MHz}$. The final $90^{\circ}$ tomography pulse occurs $8 \mathrm{~ns}$ following the end of the bias pulse to ensure proper settling of the bias current, and it is $4 \mathrm{~ns}$ long, which is short compared with the relaxation times. Each data point was taken with 2000 statistics and is separated in time by $0.1 \mathrm{~ns}$. (b) Plot of $P_{1}$ vs $\tau_{d}$ for the three tomography pulses $90_{X}, 90_{Y}$, and $I$. (c) Projections of the reconstructed quantum state on the $x y$ and $x z$ planes of the Bloch sphere. (d) Theoretical prediction of the evolution of the Bloch vector including relaxation using $T_{1}=110 \mathrm{~ns}$ and $T_{2}^{*}=90 \mathrm{~ns}$.

The loss is explained by the $90 \%$ measurement fidelity and a time delay of $8 \mathrm{~ns}$ between the end of the detuning and the tomography pulses resulting in an additional $\sim 10 \%$ loss. The loss due to reduced measurement fidelities can in principle be compensated by normalizing the data, but this was not done here for clarity.

This state tomography can be extended to several qubits in a straightforward manner $[12,13]$. Furthermore, given that this technique relies on single qubit rotations, the accuracy of a reconstructed quantum state is limited by the fidelity of the single qubit gates. Therefore, as the accuracy of single qubit gates improves with longer $T_{1}$ and $T_{2}$, the reliability of the reconstructed quantum state also improves.

In conclusion, we have introduced a new design for superconducting phase qubits that explicitly separates the capacitive from the inductive element of the Josephson junction. The number of TLS that couple to the qubit is reduced by an order of magnitude, improving the measurement fidelity to $90 \%$ and enabling quantum state tomography. We believe that all aspects of our qubit's performance are sufficient to demonstrate entangled states. Furthermore, our results pave a clear path towards future improvements in decoherence through the fabrication of simple capacitors with lower dielectric loss.

We acknowledge Steve Waltman and NIST for support in building the microwave electronics. Devices were made at the UCSB and Cornell Nanofabrication Facilities, a part of the NSF funded NNIN network. N. K. acknowledges support of the Rothschild Foundation. This work was supported by ARDA under ARO Grant No. W911NF-041-2004 and NSF under Grant No. CCF-0507227.

*Electronic address: martinis@ physics.ucsb.edu

[1] Y. A. Pashkin et al., Nature (London) 421, 823 (2003); D. Vion et al., Science 296, 886 (2002); E. Collin et al., Phys. Rev. Lett. 93, 157005 (2004); I. Chiorescu et al., Science 299, 1869 (2003); P. Bertet et al., cond-mat/ 0412485; A. Wallraff et al., Phys. Rev. Lett. 95, 060501 (2005).

[2] J. M. Martinis, S. Nam, J. Aumentado, and C. Urbina, Phys. Rev. Lett. 89, 117901 (2002).

[3] R. McDermott et al., Science 307, 1299 (2005).

[4] R. W. Simmonds, K. M. Lang, D. A. Hite, S. Nam, D. P. Pappas, and J. M. Martinis, Phys. Rev. Lett. 93, 077003 (2004).

[5] K. B. Cooper et al., Phys. Rev. Lett. 93, 180401 (2004).

[6] J. M. Martinis et al., Phys. Rev. Lett. 95, 210503 (2005).

[7] We use the term single-shot measurement to describe a measurement in which one out of two possible outcomes is obtained for each measurement: a 0 or 1 . The measurement fidelity describes the accuracy of correctly determining the two states.

[8] Previous work [6] showed that loss in coherence amplitude scales as $N_{c}^{2}$, where $N_{c}$ is the average number of individual TLS that couple to the qubit. The quantity $N_{c}$ scales as the junction area $A_{J}$ multiplied by the largest splitting size $S_{\max } \propto 1 / \sqrt{C}$, where $C$ is the total capacitance. Loss in coherence thus scales as $A_{J}^{2} / C$.

[9] M. Steffen, J. M. Martinis, and I. L. Chuang, Phys. Rev. B 68, 224518 (2003).

[10] I. L. Chuang, N. Gershenfeld, M. G. Kubinec, and D. W. Leung, Proc. R. Soc. A 454, 447 (1998).

[11] Y. Liu, L. F. Wei, and F. Nori, Phys. Rev. B 72, 014547 (2005).

[12] L. M. K. Vandersypen et al., Appl. Phys. Lett. 76, 646 (2000).

[13] H. Häffner et al., Nature (London) 438, 643 (2005). 